EESTI NSV TEADUSTE AKADEEMIA TOIMETISED. XIII KÖIDE FCOSIKA-MATEMAATIKA- JA TEHNIKATEADUSTE SEERIA. 1964, NR. 4

ИЗВЕСТИЯ АКАДЕМИИ НАУК ЭСТОНСКОУ ССР. ТОМ XIII

СЕРИЯ ФИЗНКО-МАТЕМАТИЧЕСКИХ И ТЕХНИЧЕСКИХ НАУК. 1964, № 4

\title{
ВХОДНОЙ ЯЗЫК ДЛЯ АВТОМАТИЧЕСКОГО ПРОГРАММИРОВАНИЯ АРИФМЕТИЧЕСКИХ ЗАДАЧ
}

\author{
М. КОТли
}

Описываемый входной язык и его транслятор для модернизированной машины М-3 используются в Институте кибернетики Академии наук Эстонской ССР.

Входной язык предназначен для программирования задач арифметического характера и не подходит для записи логических задач. Арифметические выражения записываются на входном языке в форме, близкой к обыкновенной математической записи. Используются переменные и константы двух типов: действительные и целочисленные. Действительные переменные могут иметь до трех индексов, массивы-максимальную размерность три и циклы по параметру - максимальную кратность три. В пределах одной задачи можно использовать до семи разных функций $(\sin x, \sqrt{x}$ и т. д.) одного аргумента, заданных в машинном коде и фигурирующих на входном языке как одноместные операции. Входной язык позволяет использовать подпрограммы, записанные на входном языке. Имеется возможность ввода данных с перфоленты.

Длина транслятора входного языка, составленного для нашей машины, 1934 команды. Из-за ограниченности оперативной памяти машины (1024 ячейки на ферритах и 1024 на барабане), транслятор разделен на три части, хранящиеся во внешной памяти и работающие одна за другой. Внешняя память используется при трансляции и для хранения обрабатываемой программы.

Время трансляции одной программы, которая помещается в оперативной памяти, $5-25$ мин.

Составленная программа работает при помощи специальной интерпретирующей программы. Однажды составленную программу можно использовать многократно.

Опыт применения входного языка и транслятора в Институте кибернетики показывает, что он: 1) позволяет программировать большинство неболыших инженерных задач, 2) легко и быстро усваивается потребителями машины, 3) уменьшает время, затрачиваемое для составления и отладки программ.

\section{1. Алфавит входного языка}

Алфавит входного языка состоит из 63 символов, кодируемых двухзначными восьмеричными числами. Слова, напечатанные жирным шрифтом, понимаются как единые символы. 


\begin{tabular}{|c|c|c|c|c|c|c|c|}
\hline Символ & Код & Символ & Код & Символ & Код & Символ & Код \\
\hline $\begin{array}{l}E \\
F \\
G \\
H \\
I \\
J \\
K \\
L \\
L \\
M \\
N \\
O \\
P\end{array}$ & $\begin{array}{l}01 \\
02 \\
\\
03 \\
04 \\
\\
05 \\
06 \\
07 \\
10 \\
11 \\
12 \\
13 \\
14 \\
15 \\
16 \\
17 \\
20\end{array}$ & $\begin{array}{l}0 \\
1 \\
2 \\
3 \\
4 \\
5 \\
6 \\
7 \\
8 \\
9 \\
1 \\
\text { ) } \\
\text { * } \\
; \\
\\
+\end{array}$ & $\begin{array}{l}40 \\
41 \\
42 \\
43 \\
44 \\
45 \\
46 \\
47 \\
50 \\
51 \\
52 \\
53 \\
54 \\
55 \\
56 \\
57 \\
60 \\
61\end{array}$ & $\begin{array}{c}Q \\
R \\
S \\
T \\
U \\
V \\
X \\
Y \\
Z \\
\text { блок } \\
\text { подпрограм- } \\
\text { ма } \\
\text { возврат } \\
\text { начало цик- } \\
\text { ла } \\
\text { конец цикла } \\
\text { целые }\end{array}$ & $\begin{array}{l}21 \\
22 \\
23 \\
24 \\
25 \\
26 \\
27 \\
30 \\
31 \\
32 \\
\\
\\
33 \\
34 \\
\\
\\
35 \\
36 \\
37\end{array}$ & $\begin{array}{l}\text { Функции од- } \\
\text { ного аргу- } \\
\text { мента } \\
\text { если } 0 \\
\text { если + } \\
\text { переход }\end{array}$ & $\begin{array}{r}62 \\
63 \\
64 \\
65 \\
66 \\
67 \\
70 \\
71 \\
72 \\
73 \\
74 \\
75 \\
76 \\
77\end{array}$ \\
\hline
\end{tabular}

Символы алфавита делятся на 3 группы: буквы, цифры и разделители.

I.1. Бу к в ы

$<$ буква > : : $=A|B| C|D| E|F| G|H| I|J| K|L| M|N| O|P|$

$Q|R| S|T| U|V| X|Y| Z \mid$ блок|подпрограмма |

возврат | начало цикла|конец цикла|целые

I.2. Ци фр ы

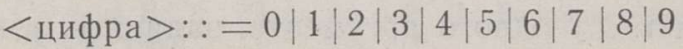

I.3. Р а здели тели

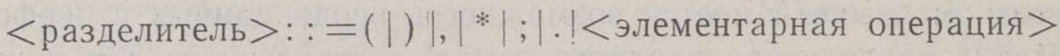

<элементарная операция $>::=<$ одноместная операция >|

$<$ двухместная операция $>\mid$ если $0 \mid$ если $+\mid$ переход $\mid=$

$<$ одноместная операция $>::=-\mid<$ функция одного аргумента $\rangle$

$<$ двухместная операция $>::=+|-| 1-|||| X$.

Функция одного аргумента есть произвольный символ (напр., $\sqrt{\text {, }}$ $\sin )$, кодируемый двухзначным восьмеричным числом из указанного диапазона.

Символ «|-|» обозначает вычитание модулей, - «/» деление и «Х»- умножение.

\section{2. Слова}

Для обозначения предметов входного языка из букв и цифр образуются слова.

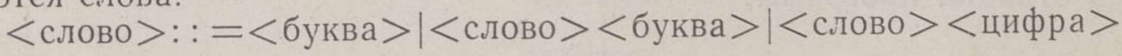

Пр и ме ры: $A$ 
Д.лина слова не ограничена, но два слова, в которых пять первых символов совпадают, воспринимаются программируюшей программой как идентичные.

Девять слов имеют фиксированное значение, и их нельзя употреблять на входном языке в другом смысле.

Это:

\begin{tabular}{|c|c|}
\hline Слово & Русский перевод \\
\hline 1. MASS & массивы \\
\hline 2. $S T P$ & стоп \\
\hline 3. $T R$ & печать \\
\hline 4. $S V$ & ВВод \\
\hline $\begin{array}{l}\text { 5. } L O P P \\
\text { 6. } I\end{array}$ & конец программы \\
\hline $\begin{array}{ll}\text { 7. } & J \\
\text { 8. } & K \\
\text { 9. } \\
\text { Челые }\end{array}$ & параметры циклов \\
\hline
\end{tabular}

Слова, начинающиеся буквами блок, подпрограмма, возврат, начало цикла, конец цикла, выделяются для обозначения разных наименований, как, например, наименования блоков, циклов, подпрограмм и т. д. Все остальные слова можно использовать для обозначения массивов и переменных.

\section{3. Числа}

$<$ число $>::=<$ целое число $>\mid<$ действительное число $>$

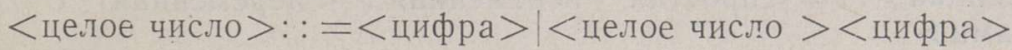

П р и ме ры: 0

1963
$<$ действительное число $>::=<$ рациональное число $>\mid<$ число с пла- вающей запятой>

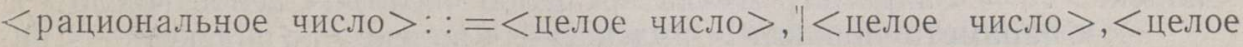 число $>$
$<$ число с плавающей запятой $>::=<$ рациональное число $>*<$ поря- док $>$
$<$ порядок $>::=<$ целое число $>\mid+<$ целое число $>\mid-<$ целое число $>$
При ме ры: 0 ,
3 ,
4,51
$34,7 *-5 \quad\left(34,7 \cdot 10^{-5}\right)$
$0,13 *+3 \quad\left(0,13 \cdot 10^{+3}\right)$

Целые числа могут иметь значения от 0 до 9999999. Рациональные числа могут иметь максимально 7 значащих цифр. Если рациональное число длиннее, то последние цифры отбрасываются. Например, число 0,00342735795 воспринимается как $0,3427357 \cdot 10^{-2}$; число 3452043400,1 kak $0.3452043 \cdot 10^{10}$.

7 ENSV TA Toimetised T-4 64 . 


\section{4. Наименования}

$<$ наименование массива $>::=\langle$ слово $>$

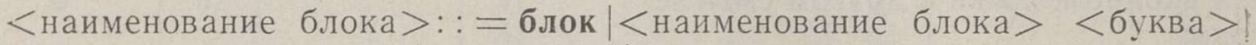

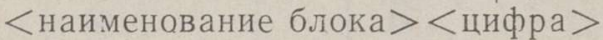

$<$ наименование цикла $>: ;=$ начало цикла $\mid<$ наименование цикла $>$

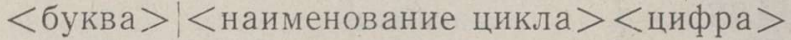

$<$ наименование подпрограммы $>::=$ подпрограмма $\mid<$ наименование

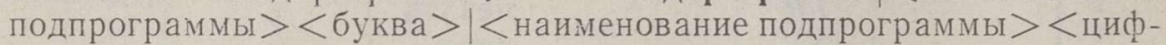
pa>

$<$ наименование возврата $>::=$ возврат $\mid<$ наименование возврата $>$

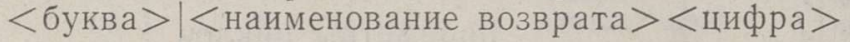

$<$ конец пнкла $>::=$ конец цикла $\mid<$ конец цикла $><$ буква $>\mid<$ конец

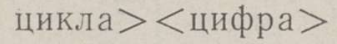

Приме ры: блок 1

начало цикла $2 A$
подпрограмма $A B$
возврат $A B$
конец цикла $2 A$

\section{5. Переменные}

$<$ переменная $>::=<$ простая переменная $>\mid<$ переменная с индексами >

$\langle$ простая переменная $>::=\langle$ слово $\rangle$

$<$ переменная с индексами $>::=<$ наименование массива $>(<$ список индексов $>$ )

$<$ список индексов $\rangle::=\langle$ индекс $\rangle \mid\langle$ индекс $\rangle ;\langle$ индекс $\rangle \mid\langle$ индекс $\rangle$; $<$ индекс $>$; <индекс $>$

$<$ индекс $\rangle::=\langle$ параметр цикла $\rangle \mid<$ целое число $\rangle \mid<$ целое число $\rangle \times$ $X<$ параметр цикла $>\mid<$ индекс $>+<$ индекс $>\mid<$ индекс $>-<$ индекс $>$

$<$ параметр цикла > : : $=l|J| K$

Пр имеры: $A$

$$
\begin{aligned}
& X 1 Y \\
& A(0 ; 1) \\
& B 1(2 \times K-1 ; 3+2 \times I-J ; 10) \\
& C(3 \times J)
\end{aligned}
$$

\section{6. Декларации}

$<$ декларация целых переменных > : = целые * <список целых переменных $>$.

$<$ список целых переменных $>::=<$ целая переменная $><<$ список целых переменных $>$; <целая переменная $>$

$<$ целая переменная $>::=<$ простая переменная > 
Пр и ме р: целые * $A 1 ; N ; M ; K I$.

Все переменные, перечисленные в декларации целых переменных, и, кроме того, параметры цикла $I, J, K$ будут принимать в программе только целочисленные значения. Все остальные простые переменные и переменные с индексами принимают действительные (в форме с плавающей запятой) значения.

$<$ декларация массивов $>::=M A S S *<$ список массивов $>$.

$<$ список массивов $>::=<$ массив с размерами $>\mid<$ список массивов $>$ $<$ массив с размерами >

$<$ массив с размерами $>::=<$ наименование массива $>(<$ список размеров >

$\langle$ список размеров $>::=<$ целое число $>|<$ целое число $\rangle ;<$ целое число $>\mid<$ целое число $>$; $<$ целое число $>$; $<$ целое число $>$

П ри м ер: $M A S S * A(10) B 1(3 ; 4) C(2 ; 5 ; 3)$.

Массивы могут быть одно-, двух- или трехмерные. Если размеры массива $m_{1}, m_{2}$ и $m_{3}$, то индексы элементов массива принимают значения $0,1, \ldots m_{1}-1 ; 0,1, \ldots m_{2}-1$ и $0,1, \ldots m_{3}-1$. Каждая переменная с индексами должна принадлежать к одному из массивов, перечисленных в декларации массивов.

Элементы массива упорядочены так, что в первую очередь изменяется последний индекс и последним - первый индекс.

Например, элементы массива $B(2 ; 3)$ будут $B(0 ; 0) ; B(0 ; 1) ; B(0 ; 2)$; $B(1 ; 0) ; B(1 ; 1) ; B(1 ; 2)$.

\section{7. Арифметические выражения}

$<$ арифметическое выражение $>::=<$ действительное арифметическое выражение $>\mid<$ целочисленное арифметическое выражение $>$

$<$ первичное выражение $>::=<$ действительная переменная $>\mid<$ действительное число $>\mid(<$ действительное арифметическое выражение $>) \mid<$ одноместная операция $><$ первичное выражение $>$

$<$ действительное арифметическое выражение $>::=<$ первичное выражение $>\mid<$ действительное арифметическое выражение $>$

$<$ двухместная операция ><действительное арифметическое выражение >

$<$ действительная переменная $>::=<$ переменная с индексами $>\mid<$ простая переменная >

Пр и мер ы: 2,

$$
\begin{aligned}
& -A 1 \\
& (2,+A 1 \times B)-3, / B \\
& \sin X+\cos V(X+A) \times B(I ; J+1)
\end{aligned}
$$

$<$ цлочисленное арифметическое выражение $>::=<$ целое число $>$ | $<$ цлая переменная $>\mid-<$ целое число $>\mid-<$ целая переменная $\rangle \mid<$ целое число или целая переменная $\rangle\langle+,-, \chi$ или $|>$ <целое число или целая переменная > 
П р и ме ры: 2

$$
\begin{aligned}
& I \\
& -N \\
& I+2 \\
& J / N
\end{aligned}
$$

где $N$ переменная, фигурирующая в декларации целых переменных. I и $J$ как параметры цикла всегда целочисленные переменные.

Старшинство операций следующее:
1. одноместная операция
2. X
3. 1
5. -
6. +
7. $=$
4. $1-1$
8. если 0 , если +

Операции одинаковых рангов выполняются в последовательности их появления в выражении.

В следующем примере отмечен порядок выполнения операции:

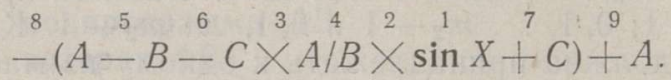

\section{8. Операторы}

$<$ оператор $>::=<$ оператор присваивания $>\mid<$ оператор перехода $>\mid$ $\mid<$ оператор ввода $>\mid<$ оператор вывода $>\mid<$ оператор останова $>\mid$ $<$ оператор обращения к подпрограмме >

8.1.

$<$ оператор присваивания $>::=<$ действительная переменная $>=<$ дей-

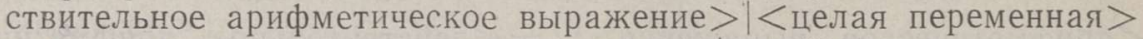
$=<$ целое арифметическое выражение $>\mid<$ действительная пере-

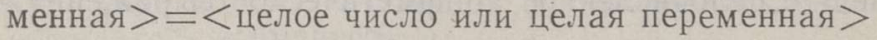

Последняя форма оператора присваивания предусмотрена для перехода от целочисленных величин к действительным.

$$
\begin{aligned}
\text { Примеры: } & A 1=-B \times \sin (X-A) \\
& I=J \times K \\
& B 1(I ; J ; K-1)=B 1(I ; J ; K)-2, \times A \\
& A=J .
\end{aligned}
$$

Целочисленными здесь являются $I, J, K$.

8.2 .

$<$ оператор перехода $>::=<$ оператор условного перехода $>\mid<$ оператор безусловного перехода $>$

$<$ оператор условного перехода $>::=<$ арифметическое выражение $>$ $<$ операция условного перехода $><$ наименование блока, цикла или возврата $>\mid<$ оператор присваивания для действительной переменной $><$ операция условного перехода $><$ наименование блока, цикла или возврата >

<операция условного перехода > : = если $0 \mid$ если + 
Пр и ме ры: $I$ если 0 блок $A$

$$
\begin{aligned}
& K-J \text { если }+ \text { возврат } 2 \\
& Y=\sin X-B \text { если }+ \text { начало цикла } C
\end{aligned}
$$

$<$ оператор безусловного перехода>: : = переход<наименование блока, цикла или возврата>

Пр им еры: - переход блок $A$ переход возврат $C 1$

8.3 .

$<$ оператор останова $>::=S T P \mid S T P<$ номер останова $>$

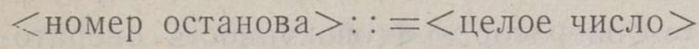

8.4.

$<$ оператор ввода $>::=S V^{*}<$ переменная $>\mid S V^{*}<$ переменная с индексами $><$ переменная с индексами $><ш а г>$ $<$ шаг $>::=<$ целое число $>\mid<$ целая переменная $>$

Пр и меры: $S V^{*} I$

$$
\begin{aligned}
& S V * A 1(I ; J ; K) \\
& S V * A(0 ; 0) A(9 ; 9) 1 \\
& S V * B(0 ; 0) B(J ; 0) 5
\end{aligned}
$$

Оператор ввода первого типа вводит с перфоленты одно число и присваивает его значение указанной переменной. Оператор ввода второго типа вводит с перфоленты массив чисел и присваивает их значения элементам массива, первый и последний элемент, которого указаны. Шаг указывает шаг движения по массиву. Например, в случае третьего примера, если размеры массива $A m_{1} \geqslant 10$ и $m_{2}=10$, то вводятся

$$
\begin{aligned}
& A(0 ; 0) A(0 ; 1) \ldots A(0 ; 9) \\
& A(1 ; 0) A(1 ; 1) \ldots A(1 ; 9) \\
& A(9 ; 0) A(9 ; 1) \ldots A(9 ; 9) .
\end{aligned}
$$

В случае четвертого примера вводятся

$$
B(0 ; 0) B(1 ; 0) B(2 ; 0) \ldots B(J ; 0),
$$

если размеры массива $B$ есть $m_{1} \geqslant J$ и $m_{2}=5$.

8.5 .

$<$ оператор вывода $>::=T R *<$ переменная $>\mid T R *<$ переменная с индексами $><$ переменная с индексами $\rangle\langle$ шаг $\rangle$

$\Pi$ римеры: $T R * K$

$$
\begin{aligned}
& T R * A(J ; K ; 0) \\
& T R * A(0 ; 0 ; 0) A(1 ; 3 ; 5) 1 .
\end{aligned}
$$

Оператор вывода печатает значение указанной переменной или значения элементов массива, первый и последний из которых указаны. Шаг означает шаг движения по массиву. 
8.6.

$<$ оператор обращения к подпрограмме $>::=<$ наименование подпрограммы>

П р и ме р: подпрограмма $A B$.

\section{9. Блоки}

$<$ блок $>::=<$ наименование блока $>*<$ совокупность операторов $>$. $<$ совокупность операторов $>::=<$ оператор $>\mid<$ совокупность операторов $>$; <оператор $>$

Пример: блок $1 * Y=A$;

$$
\begin{gathered}
B=Y+C / 2, \\
N 1=\cos B \text { если }+ \text { блок } B ; \\
T R * N 1 ; \\
S T P .
\end{gathered}
$$

\section{0. Циклы по параметру}

$<$ цикл $>::=<$ заголовок цикла $><$ совокупность блоков $><$ конец цикла>

$<$ заголовок цикла $>::=<$ наименование цикла $>$ ( $<$ параметр цикла $>$; <начальная величина параметра > ; <шаг параметра > ; <конечная величина параметра $>$ )

$<$ начальная величина параметра $>::=<$ целое число $>\mid<$ целая переменная >

$<$ шаг параметра $>::=<$ целое число $>\mid<$ целая переменная $>$

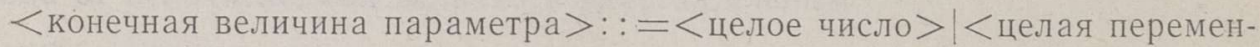
ная>

$<$ совокупность блоков $>::=<$ блок $>\mid<$ цикл $>\mid<$ совокупность блоков $><$ блок $>\mid<$ совокупность блоков $><$ цикл $>$

П р и ме р: начало цикла $A(I ; 0 ; H ; N)$

блок $1 * A(I)=X+\sin Y-B(I-1)$.

начало цикла $B(J ; 1 ; 2 ; I)$

блок $B * C(I ; J)=A(I) \times A(J) ; T R * C(I ; J)$.

конец цикла $B$.

конец цикла $A$.

$H$ и $N$ в примере целочисленные переменные, декларированные раньше.

Так как для обозначения параметров цикла выделено только три леременных $I, J$ и $K$, то можно образовать одно-, двух- или трехкратные циклы. Если кратность циклов больше трех, то нужно образовать некоторые циклы другими средствами, например при помощи счетчиков. 


\section{1. Подпрограммы}

$<$ подпрограмма $>::=<$ список деклараций $><$ наименование подпрограммы $>*<$ совокупность блоков $><$ наименование возврата $>$

$<$ список деклараций $>::=<$ пусто $>\mid<$ список деклараций $><$ декларация >

$\Pi$ р и м ер: целые *N.MASS*A (20).

подпрограмма $A B *$ блок $A$ *
блок $B * \ldots ; \ldots ; \ldots$
начало цикла $1(I ; 0 ; 1 ; N)$
блок $1 * \ldots ; \ldots ; \ldots \ldots$
блок $2 * \ldots ; \ldots ; \ldots$
конец цикла 1.
блок $C * \ldots ; \ldots ; \ldots$
возврат $A B$.

\section{2. Программа}

$<$ программа $>::=<$ список деклараций $><$ совокупность подпрограмм $><$ совокупность блоков $>L O P P$.

$<$ совокупность подпрограмм $>::=<$ пусто $\rangle \mid<$ совокупность подпрограмм $><$ подпрограмма $>$

П р и м ер: целые * $M ; N$.

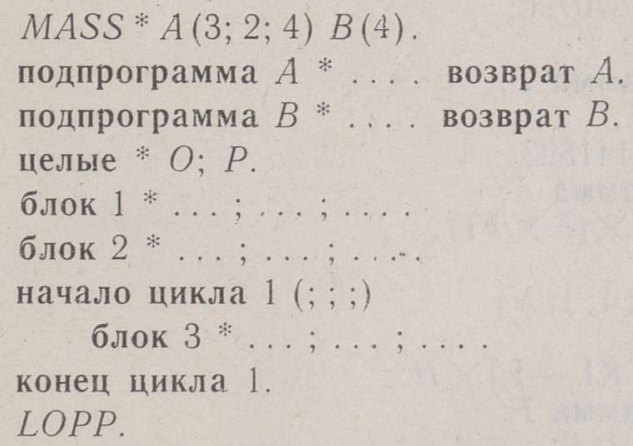

В программе все подпрограммы должны стоять перед основной проэраммой. При этом в каждой подпрограмме можно обращаться к подпрограммам, стоящим как впереди, так и за этой подпрограммой. Декларации массивов и целых переменных должны стоять перед подпрограммой или программой, в которой первый раз используются элементы, перечисленные в декларациях массивов или целых переменных.

\section{3. Примеры программ}

а) Скалярное произведение векторов

C перфоленты вводятся уменьшенная на единицу длина векторов $K<30, K+1$ элементов вектора $A$ и $K+1$ элементов вектора $B$. Скалярное произведение печатается. 


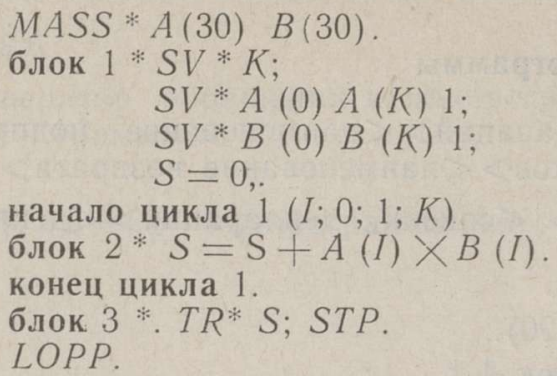

б) Вычисление интеграла

$$
J(R)=\int_{0}^{\pi} \frac{d V}{R^{2}-2 R \cos V+1}
$$

для $R=0,01 ; 0,02 ; \ldots ; 0,99$ по методу Симпсона (см. Автоматизация программирования, M., 1961, стр. 330). С перфоленты водится увеличенная в три раза точность $E P S$. Для каждого значения параметра $R$ печатаются значения параметра и интеграла.

целые * $N$.

подпрограмма $F$ *

блок $F * F=1, /(R \times R \rightarrow 2, \times R \times \cos V+1$,$) .$

возврат $F$.

блок 1 * $S V * E P S$;

$R=0,01$.

блок 2 * IRMAX $=4, / R$;

$N=1$

$H=1,570796$;

$V=0$;

подпрограмма $F$;

$F 1=F$

$V=3,141593$;

ধодпрограмма $F$;

блок $3 * S=0$,

$$
Y=H \times(F \times F 1) \text {. }
$$

начало цикла $1(K ; 1 ; 1 ; N)$

блок $4 * K 1=K$;

$V=(2, \times K 1-1) \times$,$H ;$

подпрограмма $F$;

$S=S+F$.

конец цикла 1.

блок 5 * $I R=Y+4, \times H \times S$;

$E P S|-|(I R-I R M A X)$ если + блок 6;

$I R M A X=I R$

$Y=(I R+Y) / 4$

$N=2 \times N$

$H=0,5 \times H$;

переход блок 3 .

блок $6 * I R=I R / 3$; $T R * R$

$T R * I R$

Ннститут кибернетики

$R=R+0,01$

$0,995-R$ если + блок 2 ; STP.

LOPP. 


\section{SISENDKEEL ARITMEETILISTE ULESANNETE AUTOMAATSEKS PROGRAMMEERIMISEKS}

\section{Kotli \\ Resümee}

Esitatakse ENSV TA Küberneetika Instituudis kasutatava programmeeriva programmi sisendkeel arvuti $M-3$ jaoks.

Sisendkeel võimaldab aritmeetilist laadi ülesandeid kirja panna harilikule matemaatilisele kirjutusviisile lähedasel kujul.
Eesti NSV Teaduste Akadeemia
Küberneetika Instituut
Saabus toimetusse
8 . II 1964

\section{PROGRAMMING LANGUAGE FOR AUTOMATIC PROGRAMMING OF ARITHMETICAL PROBLEMS}

M. Kotli

Summary

In this article a programming language for computer $M-3$ used by the Institute of Cybernetics (Academy of Sciences of the Estonian S.S.R.) is presented.
Academy of Sciences of the Estonian S.S.R., Institute of Cybernetics

Received

Feb. 8th, 1964 